\title{
Autopsia a la democracia venezolana. Tres momentos quellevaron a la muerte del orden democrático
}

\author{
Luciano Mondino*
}

\section{Resumen}

El presente trabajo tiene como objetivo comprender el nuevo fenómeno de la cuestión venezolana en el ámbito regional e internacional a partir de la identificación de los elementos que desmantelaron la democracia y obligaron a cerca de cuatro millones de venezolanos a deber exiliarse.

Palabras clave: democracia, chavismo, fases, régimen.

Autopsy to Venezuelan democracy: three moments to the death of the democratic order

\section{Abstract}

This article talks about the new phenomenon of the Venezuelan issue in the regional and international sphere from the identification of the elements that dismantled democracy and forced about four million Venezuelans to exile.

Keywords: democracy, chavismo, period, regime.

* Licenciado en Ciencia Política y Relaciones Internacionales de la Universidad Católica de La Plata. Actualmente es asesor parlamentario en la Cámara de Diputados de la Provincia de Buenos Aires, Argentina. Correo electrónico: lucianomondino@gmail.com

(iD) https://orcid.org/0000-0001-6303-2665 


\section{Introducción}

El concepto de Democracia es uno de los más importantes en la Ciencia Política porque el devenir histórico ha demostrado que en los Estados donde las democracias se han consolidado, se ha alcanzado mejores perspectivas de progreso y se han creado poderes públicos lo suficientemente robustos como para percibir gérmenes disruptivos que puedan atentar contra el orden democrático de un país. Es por eso que, si las instituciones públicas venezolanas hubieran sido lo suficientemente sólidas, ya en la década de 1990 la democracia en Venezuela hubiera advertido ciertos elementos que hubieran permitido dilucidar que finalmente el gobierno de Chávez degeneraría en un cuestionamiento al sistema político tradicional y podría en jaque casi la totalidad de las instituciones.

La democracia en Venezuela ha sido observada desde los últimos años producto de decisiones arbitrarias del gobierno de Chávez en pos de construir una democracia que defienda los intereses de los venezolanos y no de las elites. Sin embargo, desde la asunción de Nicolás Maduro se ha incrementado la cantidad de venezolanos que han emigrado de su país escapando de un régimen que ha asfixiado a los poderes públicos y que ha avanzado contra cualquier tipo de libertad individual.

Esta consecuencia del exilio forzoso es para América Latina y el Caribe un fenómeno importante y novedoso ya que implica que los países más cercanos como Brasil y Colombia en mayor medida, pero Argentina, Uruguay y Chile en menor, estén preparados para recibir a estos venezolanos que ocupan, según la ACNUR, la calidad de refugiados.

Considerando entonces estos elementos preliminares que dejan ver la importancia de los regímenes democráticos como canales para el progreso y desarrollo de los países, este trabajo tiene como objeto identificar tres períodos en el desmantelamiento de la democracia en Venezuela que explican la situación actual y que permite identificar la problemática de los refugiados venezolanos no solamente al ámbito nacional sino también al ámbito regional a partir de la OEA y al internacional a partir de la ONU y su reciente denuncia a la violación de los derechos humanos.

El primer período que se sitúa a finales de la década del noventa es titulado como la etapa embrionaria del desmantelamiento de la democracia a partir de la cual pueden identificarse elementos antidemocráticos en formación que hubieran permitido advertir cuál era el proyecto chavista que estaba en gestación.

Estos elementos que han sido variados y que se han caracterizado por actos ilegales de cuestionamiento al orden democrático, han sido el puntapié de un proyecto chavista de reconfigurar la democracia como era conocida comúnmente para dar lugar a una 
épica discursiva de defensa de los intereses venezolanos y en contra de los intereses de las elites venezolanas y mundiales. Digo épica discursiva porque paradójicamente la vinculación con los Estados Unidos o entre George Bush y Hugo Chávez ha sido de confrontación en el discurso, pero de acercamiento en lo económico en torno al petróleo.

El segundo período que abarca entre 2003 tras el referéndum revocatorio y el año 2013 es una década que contiene diferentes hechos que permiten visibilizar un desmantelamiento progresivo de la democracia al punto de herirla casi de muerte. Es el período de la muerte de la democracia porque aquí el gobierno venezolano logró modificar las reglas de juego a su favor y ha cristalizado una crítica sistémica no solo al sistema político tradicional sino también al sistema de partidos principalmente opositores quienes fueron deslegitimados.

El año 2003 lo considero como un parteaguas en la progresiva fractura del orden democrático porque es a partir de dicho referéndum que se produce una batalla entre el oficialismo y la oposición. A partir de aquí el gobierno optó por la consolidación de una dicotomía amigo-enemigo.

El tercer y último periodo es el de la implosión del sistema económico y la confrontación extrema con la mayoría de los países del mundo por el reconocimiento, finalmente, por parte de la ONU sobre la violación de los derechos humanos en Venezuela: la falta de libertades políticas, civiles y los presos políticos del chavismo.

\section{La democracia en términos reales}

Desde los pensadores clásicos hasta la actualidad, se han desarrollado diversos análisis de los elementos constitutivos de la democracia y se ha variado dependiendo del uso que adopte o del contexto en el cual se utilice. En este sentido, científicos clásicos de la ciencia política como Norberto Bobbio (1984) han advertido que entender la democracia como «el gobierno de todos» es un ideal límite ya que «incluso en cualquiera de los regímenes más democráticos no votan todos los individuos sino los que han alcanzado cierta edad» (p. 14). Pero también Bobbio afirma «que los derechos y las libertades tales como la libertad de opinión, de expresión de la propia opinión, de reunión, de asociación, etc., deben estar garantizados» (p. 15) para poder producir un acercamiento más certero a lo que conocemos como el ideal democrático.

Por otro lado, Giovanni Sartori (1993) define lo que no es la democracia con una «lista de términos utilizables como opuestos de democracia variada: tiranía, despotismo, dictadura, absolutismo, autoritarismo, totalitarismo y autocracia» (p. 118). Analizar cada uno de estos conceptos depararía una desviación original de este trabajo y es 
por eso por lo que se prefiere optar por la dictadura dentro de la valoración que hace Sartori para explicar lo que no es la democracia: La democracia degenera en dictadura cuando no se han alterado los elementos básicos que la constituyen.

Sartori (1993) entonces define a la dictadura como «una forma de Estado y una estructura del poder que permite su uso ilimitado (absoluto) y discrecional (arbitrario)» (p. 129). Podemos afirmar entonces que la democracia va a ser una forma de Estado en el cual se repele el uso ilimitado y discrecional del poder en pos de proteger la regla de las mayorías garantizando los derechos y las libertades.

Finalmente, el aporte de Juan Linz (1978) ha permitido definir a la democracia como la «libertad legal para formular y proponer alternativas políticas con derechos concomitantes de libertad de asociación, expresión y otras libertades básicas de la persona; competencia libre y no violenta entre líderes con una revalidación periódica de su derecho para gobernar; inclusión de todos los cargos políticos efectivos en el proceso democrático y participación de todos los miembros de la comunidad política, cualesquiera que fuesen sus preferencias políticas» (Linz, 1978, p. ???).

Construir el concepto de democracia a partir de diversos autores permite hallar elementos constitutivos en común tales como la defensa de las libertades básicas que recaen sobre el hombre y la mujer, la limitación del poder absoluto y discrecional como así también la necesidad de instaurar regímenes democráticos para consolidar mayores y mejores proyectos de nación.

Sin embargo, en simultáneo con la construcción teórica del concepto, la democracia en términos reales implica una complejidad mayor en la medida que los Estados, principalmente latinoamericanos y caribeños, poseen estructuras institucionales débiles que impiden la percepción de estos elementos autoritarios cuando se encuentran en las etapas formativas. Esta debilidad tiene sus causas principalmente en los altos grados de corrupción, los bajos estándares de transparencia, el casi nulo acceso a la información pública y la ausencia de auténticos gobiernos abiertos que fomenten la participación ciudadana.

El caso de Venezuela ha sido el más significante considerando la repercusión mundial que ha tenido el hecho de que poco más de cuatro millones de venezolanos debieron abandonar su tierra natal buscando un mejor futuro. En cifras recientes, la ACNUR (2019) ha determinado que 4054870 de venezolanos y venezolanas han debido abandonar sus hogares por la agobiante crisis económica, política y social producida por el quiebre democrático.

Para intentar considerar una definición propia de la democracia consensuando las aproximaciones propuestas a partir de los autores citados anteriormente, la democracia es 
el principio por el cual se salvaguarda la regla de las mayorías garantizando los derechos y libertades para repeler el uso ilimitado y discrecional del poder; $y$ fomentar el desarrollo y la prosperidad de un Estado. En las siguientes tres fases se podrá percibir cómo el chavismo ha producido un gradual desmantelamiento de la democracia que se ha inclinado a la represión y la persecución de los políticos disidentes en la medida que el modelo político chavista fue entrando en crisis por diversos factores.

\section{La primera fase: el germen disruptivo en la etapa embrionaria}

La etapa embrionaria comprende los años transcurridos entre 1992 con los primeros alzamientos militares contra el gobierno constitucional hasta 1998 que es la consumación de las elecciones por las que Chávez terminó accediendo a la presidencia con un respaldo de $56 \%$ de los votos.

La particularidad de estos ańos es que sucedieron una serie de actos ilegales dirigidos con la intención de deslegitimar a la democracia venezolana, el sistema de partidos tradicionales —encabezado en el Polo Democrático- y el sistema político en general ya que, según Chávez, estaba hecho a medida de los intereses de las elites.

Por otra parte, la crítica sistémica esbozada por el chavismo durante estos ańos respondió también a una crítica al sistema internacional que para ese entonces se estaba conformado en torno a la caída del Muro de Berlín, el triunfo del capitalismo por sobre el comunismo y la resistencia en el Foro de São Paulo. Del mismo modo que Chávez consideraba que la democracia venezolana era únicamente hecha en función de los intereses de las elites; también creía que Venezuela en algún momento sería pisoteada por los intereses imperialistas. En este sentido la vinculación con George Bush fue tirante desde el principio, aunque posteriormente no haya habido instancias de negociaciones principalmente comerciales en torno al petróleo.

Respecto al rechazo del sistema de partidos políticos, las críticas iban especialmente dirigidas hacia los partidos tradicionales como la Acción Democrática y el Comité de Organización Política Electoral Independiente (COPEI).

Estas críticas sistémicas propias de la ideología sostenida por Chávez dieron lugar a que en 1992 un grupo de las Fuerzas Armadas comandados por Hugo Chávez Frías intentaran un levantamiento armado a fin de terminar con el gobierno de Carlos Andrés Pérez de la Acción Democrática. En este sentido cabe remarcar ambos intentos de golpes de estado fallidos no solamente porque representaron el primer acto violento contra la democracia venezolana fuertemente cuestionada en la etapa formativa del chavismo, sino también porque sería un antecedente de peso a la hora de diagramar el nuevo esquema de poder a partir del ańo 2000: las Fuerzas Armadas 
comenzarían a tomar cada vez mayor influencia en la toma de decisiones públicas y se transformarían en un actor trascendente para la política chavista.

Sin embargo, ambos levantamientos de 1992 se dieron en un contexto de debilidad institucional ya que el gobierno de Pérez transitaba para ese entonces un proceso de desgaste en comparación con su primer gobierno que había sido el de la Venezuela Saudita por las bonanzas alcanzadas por el precio internacional del petróleo y el del mayor PBI per cápita en la historia venezolana. Este segundo gobierno de Pérez estaba dentro de lo que Chávez consideraría cuatro años más tarde como «el modelo rentístico y de dependencia que seguía los lineamientos de Ronald Reagan y que en última instancia terminaban perjudicando a los trabajadores y los sectores más rezagados» (Chávez, 1996).

Los altos niveles de impopularidad y el impacto de políticas de ajuste detonaron una reacción violenta por parte de por parte de los sectores más vulnerables de la sociedad venezolana en lo que se conoció como el Caracazo de 1989 y que inauguró un período de inestabilidad política en la medida que para los venezolanos, estos partidos tradicionales no podían dar respuestas a las demandas sociales que se planteaban. Este fue el terreno propicio para que la figura de Chávez tomara cada vez más mayor protagonismo y que, hacia el 1992, se hiciera conocido por encabezar un levantamiento armado.

Luego de haber sido sofocado por las fuerzas del gobierno nacional y haber permanecido dos años en prisión, Chávez inició un proceso de intento de legitimación de sus actos para dotarlos de una mayor legalidad. Esto puede entenderse como un cambio en la visión del líder al comprender que el escenario a partir de la inestabilidad institucional y económica podía dejar lugar a que su figura emerja como la alternativa a la clase política tradicional.

Aquí merece atención un último aspecto respecto del resto de los partidos y los políticos tradicionales: Fueron muy pocos quienes creyeron que la figura de Chávez y su proyecto chavista llegaría a buen puerto. Muchos de ellos creyeron que el proyecto no tendría cabida en las urnas y que la base programática estaba destinada al fracaso. El devenir histórico demostró todo lo contrario y dejó en evidencia la falta de instituciones lo suficientemente sólidas que hubieran permitido el ascenso de Hugo Chávez a la presidencia.

Respecto a la construcción programática e ideológica del embrionario chavismo, la Agenda Alternativa Bolivariana fue mucho más que una mera presentación política de un candidato a presidente que aspiraba ganar las elecciones de 1998: en este documento el Movimiento Bolivariano Revolucionario definió ahora al sistema político venezolano como «un modelo impuesto que ya ha muerto», que «siempre se ha 
basado en la explotación, la dominación y el exterminio» y que se ha caracterizado por mantener un modelo rentístico, de explotación del petróleo y de dependencia (Chávez Frías, 1996).

La base programática del chavismo esconde una contradicción que es posible percibirla 23 años después de su elaboración y es que el modelo económico productivo del chavismo terminó consolidando un modelo igual o más dependiente del petróleo como recurso único para la economía venezolana. Esto queda manifiesto cuando a partir de 2013 y 2014 el precio internacional del barril de petróleo comenzaría a descender y haría crujir en más de una ocasión a un modelo obsoleto que no obtendría ninguna posibilidad de salvación por no haber diversificado su recurso explotado.

Finalmente, en 1998, Hugo Chávez fue electo presidente de Venezuela y en palabras de una ciudadana del estado natal del comandante «la democracia está infectada y Chávez es el único antibiótico que tenemos» (Jones, 2007, p. 225). Este testimonio resume lo característico del chavismo embrionario: una convivencia nada pacífica entre los actos ilegales y violentos con el fin de deslegitimar de origen a la democracia venezolana, el sistema político y el sistema de partidos. A partir de allí Chávez y sus seguidores más cercanos buscarían la construcción un tanto épica de que el chavismo sería el antídoto para un sistema enfermo que estaba fuertemente cuestionado.

En este momento la historia venezolana pudo haber sido distinta si la alternativa a la Acción Democrática hubiera provenido desde adentro del sistema sin recaer en un actor crítico o antisistémico como Chávez. Sin dudas el hecho de que las elecciones de 1998 arrojaran una polarización casi completa entre Chávez y Salas Romer implicaba que a partir de aquí el chavismo iba a construir el poder a partir de la crispación y la confrontación con una oposición que se anclaba en torno al $40 \%$.

\section{La segunda fase: el gobierno de Hugo Chávez, ¿̇reinterpretación de la democracia o desmantelamiento del orden?}

El 6 de diciembre de 1998 Hugo Chávez ganó las elecciones presidenciales derrotando al Polo Democrático que representaba, en la épica chavista, un polo que reflejaba a la política tradicional que no podía dar respuesta a las demandas sociales y que debía dar lugar a una nueva forma de democracia. Triunfó finalmente aquel candidato antisistema ignorado por el establishment y por los círculos de poder convencionales que desestimaron desde un comienzo este proyecto.

«Hugo Chávez era un político marginal que clamó en contra de lo que él describía como una élite gobernante corrupta y prometió construir una democracia más auténtica» (Levitsky y Ziblatt, 2018, p. 11). Pudiendo identificar los elementos 
ilegales que caracterizaron la primera etapa de desmantelamiento de la democracia, puedo afirmar que el proyecto chavista comenzaba la década del año 2000 con la puesta en marcha de un proyecto de reconfiguración de la democracia venezolana para ir hacia un nuevo modelo económico, social y político. La característica que iba a predominar durante el gobierno de Chávez es la concentración de un poder excesivo en la figura del presidente que comenzaría a fagocitar progresivamente las instituciones democráticas utilizando como medio la épica discursiva y retórica de estar construyendo una nueva democracia que ahora estaría en función de los intereses nacionales y no de los intereses burgueses, capitalistas o de las elites.

¿Cómo se puede identificar los elementos característicos que demuestren el quiebre de la democracia durante el gobierno de Chávez? Es a partir de la lectura de Juan Linz (1987) y retomado por Levitsky y Ziblat (2018) que pueden establecerse señales claras en la conducta de Hugo Chávez para afirmar que entre 2003 y 2013 se produjo un fuerte debilitamiento del orden democrático venezolano que degeneró en un proceso mucho más cruento dirigido por Nicolás Maduro.

En primer lugar, se evidencia un rechazo de las reglas democráticas o una débil defensa de las mismas en pos de construir un proyecto de democracia chavista. En esta primera instancia, Chávez rechazó o expresó la voluntad de no acatar cualquiera de las instancias legales como constitucionales, referéndum u otro mecanismo creíble y aceptable.

El primer paso hacia el autoritarismo que destaco fue a partir de 2003 al suspender un referéndum organizado por la oposición aplazándolo hasta el año entrante lo cual demostró un rechazo a las reglas del juego democrático en beneficio del gobernante. Lo curioso de esta suspensión es que el referéndum en cuestión era de tipo consultivo, es decir no vinculante, por lo cual Chávez no estaba obligado a actuar como si fuese un referéndum revocatorio que sí hubiera forzado la renuncia del presidente.

A partir de las firmas recolectadas por la oposición en el «referéndum revocatorio» se publicó la Lista Tascón, un listado entregado por la Cámara Nacional Electoral que contenía los datos personales, la huella digital y la firma de los casi dos millones de venezolanos que habían optado por la revocación constitucional del mandatario Chávez. Lo particular de la Lista fue que no solamente sirvió para identificar a todos los que habían firmado sino también para dar lugar a expresiones tales como "firmar contra Chávez es un acto de terrorismo» (El Universal, 2004). Tanto el rechazo a la instancia del referéndum como la Lista Tascón fueron hechos que avasallaron la voluntad política disidente de los venezolanos opositores y fueron ambas instancias por las cuales el gobierno venezolano sacó nuevamente a relucir una épica de confrontación entre quienes optaban por la revocación y los «reafirmatorios». 
Con el triunfalismo chavista tras haber sorteado el referéndum revocatorio y habiendo iniciado un camino en la profundización de la crispación entre venezolanos, el gobierno dio un paso más hacia la concentración de poder. En este sentido creo conveniente analizar en un apartado la dinámica del poder: al igual que con la democracia, el chavismo tuvo como objeto la concreción de un poder alternativo o de contrapoder en términos analógicos de Manuel Castells (2009) en donde los pilares del sistema político tradicional se verían totalmente perturbados por la injerencia de factores externos: la democracia cedía cada vez más lugar a la democracia chavista y la figura del presidente comenzaba a borrar barreras que impedían el uso discrecional y arbitrario del poder. El nuevo poder ahora residía en su totalidad en un Chávez que comenzaba a fortalecerse cada día más.

La democracia chavista avasalló la salvaguarda de la regla de la mayoría, los derechos y las libertades entendidos bajo una concepción liberal. Sin embargo, ha logrado también construir una aparente defensa de una participación mayor de la ciudadanía en los asuntos públicos que es posible advertir en las infinitas construcciones discursivas que comenzaron a replicarse por todo Venezuela a lo largo del Aló Presidente.

El chavismo tuvo tres instancias en las cuales el poder finalmente se concentró de manera excesiva en la figura de Chávez: la Ley Habilitante de 2007, la reforma a la Constitución Nacional y la ideologización de la educación o la «nueva educación popular».

En primer término, la Ley Habilitante de 2007 permitió al presidente la injerencia en casi todos los ámbitos del sistema venezolano: económico, social, tributario, seguridad, defensa, infraestructura o participación ciudadana. Esta propuesta del presidente Chávez, respaldada por la Asamblea Nacional, se argumentó a partir de la victoria en las elecciones y por considerar que el pueblo había optado por dicha transformación. En este sentido, la diputada Cilia Flores no dudó en catalogar a este proceso como «un mandato del pueblo que requería la adaptación de la legislación nacional al proyecto propuesto por el mandatario Chávez» (Asamblea Nacional, 2007).

En esta ley se puede visibilizar que la concentración del poder implicó una eliminación de cualquier disidencia política en los grupos opositores ya que el modelo del chavismo "había sido respaldado en las urnas» y ahora debía avanzarse en las reformas necesarias para profundizar esa nueva democracia. Nuevamente está presente la deslegitimación de origen al orden democrático, al sistema político y al de partidos en particular, pero también a cualquier expresión alternativa que pueda confrontar con la propuesta chavista: Desde 2007 la democracia chavista, como fue bautizada por los seguidores de Chávez, no se esforzó por caer en la autocrítica, sino que siempre dio un paso decidido hacia adelante en pos de profundizar las transformaciones ya realizadas. 
En consonancia con la construcción teórica propuesta anteriormente para el concepto de democracia, puedo afirmar que ya entre 2003 y 2007 se produjo una violación al principio de respeto de regla de la mayoría que no permite garantizar los derechos civiles y políticos básicos para repeler el uso ilimitado y discrecional del poder: la Asamblea Nacional no solamente dejó de funcionar como órgano de control al poder chavista, sino que fue quien posibilitó la Ley Habilitante.

La segunda de las instancias por la cual las reglas de juego democráticas se erosionaron durante el gobierno de Chávez fue la reforma constitucional, algo tan fundamental para el ideario chavista: el nuevo poder ahora no solamente residía exclusivamente en el presidente quien obtenía el control de casi la totalidad de los estamentos de la sociedad, sino que de manera indefinida Chávez podía ser reelecto si el pueblo así lo decidía. Otra gran paradoja si se considera que hacia 2007 y 2008 la oposición estaba fuertemente avasallada.

Por último, haber logrado la reforma constitucional fue haber logrado plasmar institucionalmente el proyecto embrionario trazado en la década del noventa y poder triunfar definitivamente contra quienes sostuvieron que el experimento chavista era de poco alcance.

A los efectos de dar un nuevo marco constitucional al proyecto chavista $-\mathrm{y}$ no de adecuar el proyecto a las reglas establecidas por la democracia venezolana- el presidente Chávez nombró el Consejo Presidencial para la Reforma Constitucional formado por once miembros y comenzó así a dar vida a un proyecto que prometía derrumbar cualquier tipo de estructura establecida.

Sin embargo, debe hacerse una diferencia con la reforma constitucional adoptada en 1999 y tiene que ver específicamente con el concepto de moderación propio de los primeros años del mandatario en el poder y otro contexto propio de crispación que tuvo, como se dijo, el detonante a partir de 2003 y 2004 por el referéndum revocatorio. La diferencia más clara se encuentra en que durante el período de finales de la década de 1990, el chavismo utilizó el concepto de "democracia participativa» como una respuesta al "excesivo poder de las elites tradicionales» y como una forma de poder simplificar las soluciones fomentando una mayor participación ciudadana. El contexto de la segunda reforma constitucional se identifica por la crispación el de conflicto no solamente por la disputa entre el oficialismo y la oposición —que se replicaba en muchos aspectos de la vida cotidiana venezolana- sino también porque a partir de 2001 y 2002 se promueven una importante cantidad de leyes que tińen a este período como antineoliberal y antiimperialista ubicando a los Estados Unidos como el principal insumo de confrontación: Entre estas leyes que marcan la tendencia de confrontación en el plano de política interior como de política exterior 
podemos destacar la Ley de Hidrocarburos en donde se priorizaba la figura estatal en las compañías petroleras principalmente mixtas; la Ley de Seguridad Social a partir de la cual se rechazaba cualquier intento de privatización de la misma; o la Ley de Tierras en donde se defendía el uso nacional las tierras subutilizadas.

El último elemento que considero transversal a los años de gobiernos de Chávez y que ha configurado una transformación radical en su concepción clásica, es la educación devenida en educación popular que fue el vehículo que el presidente Chávez precisó para aumentar su dominio sobre la cultura venezolana. En la nueva geometría del poder esbozada a partir del nuevo proyecto, la educación cumplía un rol esencial porque para ser el vehículo revolucionario debía dejar atrás lo que el bolivarianismo identificaba como concepción burguesa y capitalista para dar lugar, al igual que con la democracia, una educación formativa en el ámbito público y privado para todos los venezolanos.

La educación popular fomentada por el chavismo en Venezuela pudo identificarse claramente a partir de dos indicadores: en primer lugar, las alocuciones presidenciales, el "Aló Presidente», en donde el liderazgo carismático propio de los populismos de Chávez Frías podía relucirse durante más de cinco o seis horas en las cuales con un tono seductor y con datos muchas veces débiles, se repasaban los principales hitos de la revolución. En segundo lugar, la penetración del marxismo fomentada durante años por José Ramón Rivero quien fuera ministro de Trabajo y respaldado por las leyes-decreto necesarias de Chávez a raíz de los superpoderes otorgados por la Asamblea Nacional (Vinogradoff, 19 de abril de 2007; Arenas y Gómez, 2006).

\section{La tercera fase: la muerte de la democracia y el colapso del régimen}

Esta última fase que comprende los gobiernos de Nicolás Maduro y se extienden hasta la actualidad, se puede caracterizar a partir de una más que crítica situación económica, a tal punto de considerarla indescriptible, y un objeto de constantes denuncias de violaciones de los Derechos Humanos por parte de diferentes gobiernos democráticos de otros países hasta por la ONU respecto al último informe presentado en 2019 (ANUDH, 2019).

Respecto a la cuestión económica de Venezuela durante 2013 y 2019 no bastaría con dedicar algunas líneas en el presente artículo, sino que es un tema que abarcaría escritos enteros abocados únicamente al análisis de la situación macro y microeconómica del país gobernado por Nicolás Maduro. A fines de seguir con la línea planteada al principio de este trabajo, considero que la crisis económica actual es el último indicador que nos permite confirmar que la fractura de la democracia y la instauración de un modelo rentístico de explotación del recurso primario atentó en contra de la 
estabilidad económica y la prosperidad del país llevando a los venezolanos a tener que padecer picos inflacionarios y concentración de su economía a niveles escandalosos, según estimaciones del FMI durante el 2018 (FMI, 2018).

La realidad actual de Venezuela seguramente hubiera sido impensada en los momentos de la Venezuela Saudita cuando el país había comenzado un camino de ascenso y mejora de sus principales indicadores. En contracara a esto, la Venezuela de 2019 presenta signos claros de deterioro no solamente en las variables macro y microeconómicas sino también una falta de capacidad de vinculación con otros actores del sistema internacional por las acusaciones de violaciones de los Derechos Humanos y la poca calidad de transparencia en sus instancias burocráticas.

Uno de los factores externos que han condicionado el modelo bolivariano de Chávez Frías y Maduro ha sido la caída del precio de barril del petróleo. Así, en 1998 según estimaciones de la OPEP el precio del barril era de unos 16 dólares para pasar en 2012 a unos 100 dólares. Esto fue en primera medida una buena noticia para el gobierno de Chávez que comenzó a trazar su modelo a partir de una economía híper centralizada en la explotación del petróleo sin desarrollo de otro sector de la economía que pudiera compensar una eventual caída del crudo. Sin embargo, luego de crisis económicas como la de 2014, tuvo lugar una baja sensible en el precio del crudo derrumbándolo cerca de los 40 dólares en 2016. Según datos oficiales del Ministerio de Petróleo el precio de julio de 2019 se ubica cerca de los 60 dólares. Cabe mencionar, además, que desde 2017 el gobierno de Nicolás Maduro ha abandonado el patrón dólar para referenciar sus precios y ha adoptado el yuan como moneda utilizando un tipo de cambio de 6,86 yuanes para calcular el precio del petróleo. Esto identifica que la relación con los Estados Unidos ha permanecido crítica especialmente desde la asunción de Donald Trump en la Casa Blanca.

La respuesta que ha encontrado el gobierno de Maduro principalmente a la caída del precio del casi exclusivo producto de la economía venezolana ha sido un paquete de medidas económicas de corte netamente proteccionistas, pero manteniendo la negativa al desarrollo de otros sectores como el agrícola. Estas medidas han incluido, entre otras, control sobre el mercado de tipo de cambio, aunque hacia mayo de 2019 se haya eliminado el Sistema de Divisas de Tipo de Cambio Complementario Flotante de Mercado (DICOM); fuerte avance del Estado por sobre la propiedad privada a través de las nacionalizaciones y un control de precios que en términos reales no ha podido detener el paso hacia una hiperinflación.

Finalmente, y a partir del poco acceso a la información pública y el bajo nivel de transparencia que el país representa en los rankings mundiales, el Fondo Monetario Internacional ha proyectado una inflación del 10000 000\% y una caída cercana al 
25\% del PBI para el año actual. El Informe de Perspectivas de la Economía Mundial que arroja estos últimos dos datos es categórico al aclarar respecto al precio del barril de petróleo que «los riesgos al alza para los precios en el corto plazo incluyen acontecimientos geopolíticos en Oriente Medio, la agitación social en Venezuela y una postura más dura de Estados Unidos frente a Irán y Venezuela» (FMI, 2019, p. 42).

El atentado del chavismo a las reglas democráticas no solamente ha impactado en la microeconomía del país, sino que además ha acotado el margen de mercados externos e inversores directos que pretendan invertir en Venezuela. En este sentido el Banco Mundial afirma que la inversión extranjera directa o la entrada neta de capital entre 1999 y 2018 se ha desmoronado de manera pronunciada con algunos repuntes: De una balanza de pagos positiva en 1995 de 6202000 millones de dólares a una negativa de 68000 millones de dólares en 2018 (Banco Mundial, 2019).

Concluyo con estos indicadores que las barreras que el chavismo ha roto han entorpecido su vinculación con el resto del mundo ya que los países tienden a comerciar cada vez menos con los países cuyas democracias se encuentran débiles ya que las instituciones y los actores políticos internos son más propensos a la corrupción y la conformación de reglas poco transparentes.

El gobierno de Nicolás Maduro fue la última de las fases en donde la democracia ya ha llegado muerta y solamente una autopsia podría permitir visualizar los elementos que han contribuido a su desaparición. La situación del país caribeño ha sido para la región toda un foco de conflicto tan importante que ha llevado a la Oficina del Alto Comisionado de la ONU para los Derechos Humanos (ANUDH, 2019) a elaborar un informe crítico en el cual se da cuenta de la violación de los derechos y las libertades de los venezolanos que se encuentran en Venezuela y que no han podido escapar de las pretensiones de un régimen que cada día parece cerrarse más como respuesta al derrumbe de un modelo que siempre encuentra una nueva oportunidad para reinventarse.

\section{Conclusiones}

En este artículo fue posible identificar los elementos que constituyeron un proceso gradual de desmantelamiento de la democracia que se inicia desde finales de la década de 1990 y que continúa hasta nuestros días con la consumación de un régimen represivo que ha atentado con la casi totalidad de derechos civiles y políticos de los venezolanos.

El concepto de democracia desde las primeras definiciones de los pensadores clásicos hasta los más actuales representa una complejidad en la ciencia política por los 
componentes que se elijan para definirla y por el contexto en el cual se analiza su aplicación. Como quedó demostrado, Venezuela como tantos otros de los países principalmente de América Latina y el Caribe, presenta estructuras institucionales débiles que no permiten detectar cualquier germen disruptivo del orden democrático. Durante la década de 1990 ninguna institución política o judicial pudo percibir en la Agenda de Alternativa Bolivariana más que una propuesta alocada de un candidato antisistema que debía ser ignorado por la clase política tradicional. De haber alertado estos indicios, quizás la historia hubiera sido distinta para Venezuela. Del mismo modo puede hablarse de Brasil con Vargas o de Argentina con Perón.

Pero aun en el caso de las instituciones más fuertes ¿cómo se podría evitar el ascenso al poder de un líder el cual fue electo mediante vías legales como Chávez en 1998? Definitivamente esta es una pregunta que puede disparar una presentación a parte. Las críticas antisistema deben considerarse lo suficientemente serias como para presentar en el fondo una deslegitimación del modelo imperante con fines mayores: en el caso de Chávez, nuevamente, ninguna institución pudo evitar que en 1992 intentara un golpe de estado y que en 1998 triunfara en las elecciones.

En segundo lugar, la consolidación de la democracia chavista no fue más que un recurso discursivo y retórico para esconder un avasallamiento de las principales estructuras institucionales de Venezuela durante casi una década. El contrapoder que Chávez ha desarrollado durante su mandato culminó con un uso ilimitado e indiscriminado del mismo muchas veces en contra de sus adversarios políticos a quien no dudó en tildar de cipayos.

Durante el gobierno de Chávez se dieron una serie de hechos que pueden identificar el proceso gradual de desmantelamiento de la democracia a partir de una alteración de los elementos que componen al concepto: Partiendo de una nula o poca defensa de las reglas de juego democráticas y una deslegitimación de origen, el gobierno de Hugo Chávez aceleró en contra de las libertades civiles y políticas a partir de una concentración enorme de poder que le permitió obtener la sanción de la Ley Habilitante, la reforma constitucional y fomentar la educación popular como vehículo para expandir la revolución chavista por toda Venezuela. Nuevamente y considerando los elementos aportados en las definiciones obtenidas tanto por Bobbio (1984), Sartori (1993) o Linz (1978), podemos afirmar que durante 2003 y 2013 se produjo un atentado en contra de las libertades básicas tales como la alternancia en el poder; el derecho de expresión; la libertad de prensa y opinión; la violación de la regla de la mayoría para dar lugar a un abuso y uso discrecional del poder.

Finalmente, durante el gobierno de Nicolás Maduro, quedó consumada la muerte de la democracia y un modelo económico social y político que fue imposible de sostener 
al momento de la caída del precio del petróleo que, como advertimos, era el recurso por excelencia del modelo rentístico chavista.

El hecho haber avasallado contra las instancias y reglas de juego democráticas no solamente impidieron la posibilidad de una voz disidente, algo obvio en las dictaduras, sino que además no permitió que los opositores al chavismo promovieran una salida alternativa que hubiera evitado el colapso económico, moral, social y político que empujó a más de cuatro millones de venezolanos a tener que partir de sus hogares.

Sin dudas que la democracia es el modo por el cual los países han logrado mejores niveles de desarrollo para sus pueblos y han conseguido en el largo plazo, mejores condiciones para comerciar y vincularse con el resto de los actores internacionales. La situación en Venezuela requiere que los gobiernos democráticos de América Latina continúen denunciando la violación de los derechos en el país caribeño y fomentando la conciencia ciudadana en el respeto a las instituciones que, si se fortalecen, son los mejores parámetros que los Estados tienen para percibir cualquier germen disruptivo que pueda atentar, en un futuro, la democracia de los países.

La región en su totalidad está expectante siguiendo los resultados del chavismo en Venezuela sobre todo a partir de que Estados Unidos definió a partir de 2017 una agenda de seguridad enfocada en un nuevo eje en donde Venezuela ocupa el lugar de Estado perturbador de la región que puede arrastrar al resto de los países a experiencias similares como las que llevó adelante el chavismo en casi dos décadas. La región debe seguir de cerca porque, como se advirtió, los gérmenes totalitarios pueden estar avanzando en silencio con la complicidad inadvertida de la mayoría.

\section{Referencias bibliografías}

ACNUR - Oficina del Alto Comisionado de las Naciones Unidas para los Refugiados. (2019). Aspectos claves del monitoreo de protección: situación Venezuela. Naciones Unidas. Recuperado de https://www.acnur.org/5d321d124.pdf

ANUDH - Oficina de las Naciones Unidas para los Derechos Humanos (2019). Informe de la Oficina de las Naciones Unidas para los Derechos Humanos sobre Venezuela insta a adoptar de inmediato medidas para detener y remediar graves violaciones de derechos. Ginebra: Naciones Unidas. Recuperado de https://www.ohchr.org/sp/newsevents/ pages/DisplayNews.aspx?NewsID $=24788 \&$ LangID $=S$

Arenas, N. (2007). Poder reconcentrado: el populismo autoritario de Hugo Chávez. Politeia, 30(39), 23-63. Recuperado de https://www.redalyc.org/pdf/1700/170018341002. pdf

Arenas, N. (2010). La Venezuela de Hugo Chávez: rentismo, populismo y democracia. Nueva Sociedad, 229, 76-93. Recuperado de https://nuso.org/media/articles/downloads/3724_1.pdf 
Banco Mundial. (2018). Inversión extranjera directa, entrada neta de capital (balanza de pagos, US\$ a precios actuales)-Venezuela. Recuperado de https://datos.bancomundial.org/ indicador/BX.KLT.DINV.CD.WD?locations=VE

Bobbio, N. (1984). El futuro de la democracia. Ciudad de México: Fondo de Cultura Económica. https://doi.org/10.33426/rcg/1984/2/877

Castells, M. (2009). Comunicación y poder. Madrid: Alianza Editorial.

Chávez Frías, H. (1996). Agenda Alternativa Bolivariana: una propuesta patriótica para salir del laberinto. Caracas.

Dorta, M., Guerra, J.y Sánchez, G. (1997). Credibilidadypersistencia de la inflación en Venezuela. Caracas: Banco Central de Venezuela. Recuperado de http://200.74.197.135/Upload/ Publicaciones/docu11.pdf

FMI - Fondo Monetario Internacional. (2019). Perspectivas de la economía mundial. En Estudios Económicos y Financieros. Recuperado de https://www.imf.org/es/ Publications/WEO/Issues/2019/10/01/world-economic-outlook-october-2019

Linz, J. J. (1978). La quiebra de las democracias. Madrid: Alianza Universidad-Ciencias Sociales.

Sartori, G. (1987). Teoría de la democracia. Madrid: Alianza Editorial.

Vinogradoff, L. (19 de abril de 2007). Chávez obligará a las empresas a dar cuatro horas semanales de marxismo. ABC Internacional. Recuperado de https://www.abc. es/internacional/abci-chavez-obligara-empresas-cuatro-horas-semanales-marxismo-200704190300-1632626209260_noticia.html

Recibido: 7 noviembre de 2019 Aprobado: 24 de agosto de 2020 